\title{
Metal-Catecholate Frameworks as Solid Basic Catalysts
}

\author{
Pieterjan Valvekens $^{1} \cdot$ Maxime Stalpaert ${ }^{1}$ Giel De Winter ${ }^{1} \cdot{\text { Dirk De } \text { Vos }^{1}}^{1}$
}

Published online: 25 August 2016

(C) Springer Science+Business Media New York 2016

\begin{abstract}
Metal catecholate frameworks, based on 2,3,6,7,10,11-hexahydroxytriphenylene and bivalent metal ions $\left(\mathrm{Ni}^{2+}, \mathrm{Cu}^{2+}, \mathrm{Zn}^{2+}, \mathrm{Mg}^{2+}, \mathrm{Ca}^{2+}, \mathrm{Sr}^{2+}\right.$ and $\left.\mathrm{Ba}^{2+}\right)$ were synthesized, characterized with powder $\mathrm{X}$-ray diffraction and $\mathrm{N}_{2}$ physisorption, and evaluated as catalysts in Knoevenagel condensation and Michael addition reactions. The large number of catecholate oxygen atoms in these materials resulted in a high catalytic activity which was shown to be a function of both the porosity of the material and the electronegativity of the metal ion.
\end{abstract}

Keywords Basic catalysis - Metal-catecholate frameworks $\cdot$ Knoevenagel condensation $\cdot$ Michael addition

\section{Introduction}

In the field of MOF catalysis, MOFs with basic sites incorporated in the lattice would typically be expected to be structures built up using $\mathrm{N}$-functionalized linker molecules [1-4]. Recent findings however have shown that this does not necessarily need to be the case. In MOFs featuring structural nitrate anions for example, these anions can be selectively decomposed to basic sites which can readily be

Electronic supplementary material The online version of this article (doi:10.1007/s11244-016-0697-5) contains supplementary material, which is available to authorized users.

Dirk De Vos

dirk.devos@biw.kuleuven.be

1 Center for Surface Chemistry and Catalysis, KU LeuvenUniversity of Leuven, Celestijnenlaan 200 F, box 2461, 3001 Louvain, Belgium used for catalysis [5]. Unfortunately, the number of stable structures featuring such structural nitrate anions is scarce, making this strategy less universally applicable. By analogy to the use of $\mathrm{N}$-functionalized linker molecules, the use of linkers with specific functional groups that could more easily be introduced in MOF lattices would therefore present a more elegant approach. An example of such a specific functional group is found in MOFs of the MOF-74 type. It was shown that both the carboxylate oxygen atoms and the phenolate oxygen atoms in these MOFs possess an intrinsic basicity [6]. The basicity of the phenolate oxygen atoms is however stronger than that of carboxylate atoms due to the higher $p \mathrm{~K}_{\mathrm{a}}$ of the phenol group and the lower $p \mathrm{~K}_{\mathrm{b}}$ of the corresponding conjugate base. This allowed the MOF-74 type MOFs to perform well as catalysts in base catalyzed reactions such as Knoevenagel condensation and Michael addition reactions. Similarly, phenolate groups were also recently found to introduce basic sites in a solid Zr-HBA catalyst $(\mathrm{HBA}=$ 4-hydroxybenzoic acid) which showed catalytic activity in the transfer hydrogenation of ethyl levulinate to $\gamma$-valerolactone [7].

A strategy to improve the catalytic activity of the MOF74 type materials would therefore be to look into materials which possess phenolate oxygen atoms in large numbers. Extended metal-catecholate frameworks or M-CATs are such materials (Fig. 1a). These materials, reported by Hmadeh et al. [8, 9], consist of bivalent metal ions $\mathrm{M}^{2+}$ $\left(\mathrm{M}^{2+}=\mathrm{Ni}^{2+}, \mathrm{Cu}^{2+}\right.$ or $\left.\mathrm{Co}^{2+}\right)$ interconnected by the linker molecule 2,3,6,7,10,11-hexahydroxytriphenylene (HHTP). The material is a stacked structure consisting of two alternating layers in which the linker, HHTP, is present in different oxidation states. The first layer is an extended honeycomb structure with hexagonal pores in which the metal ions are coordinated to two adjacent fully deprotonated HHTP linkers (Fig. 1b). Two additional water 

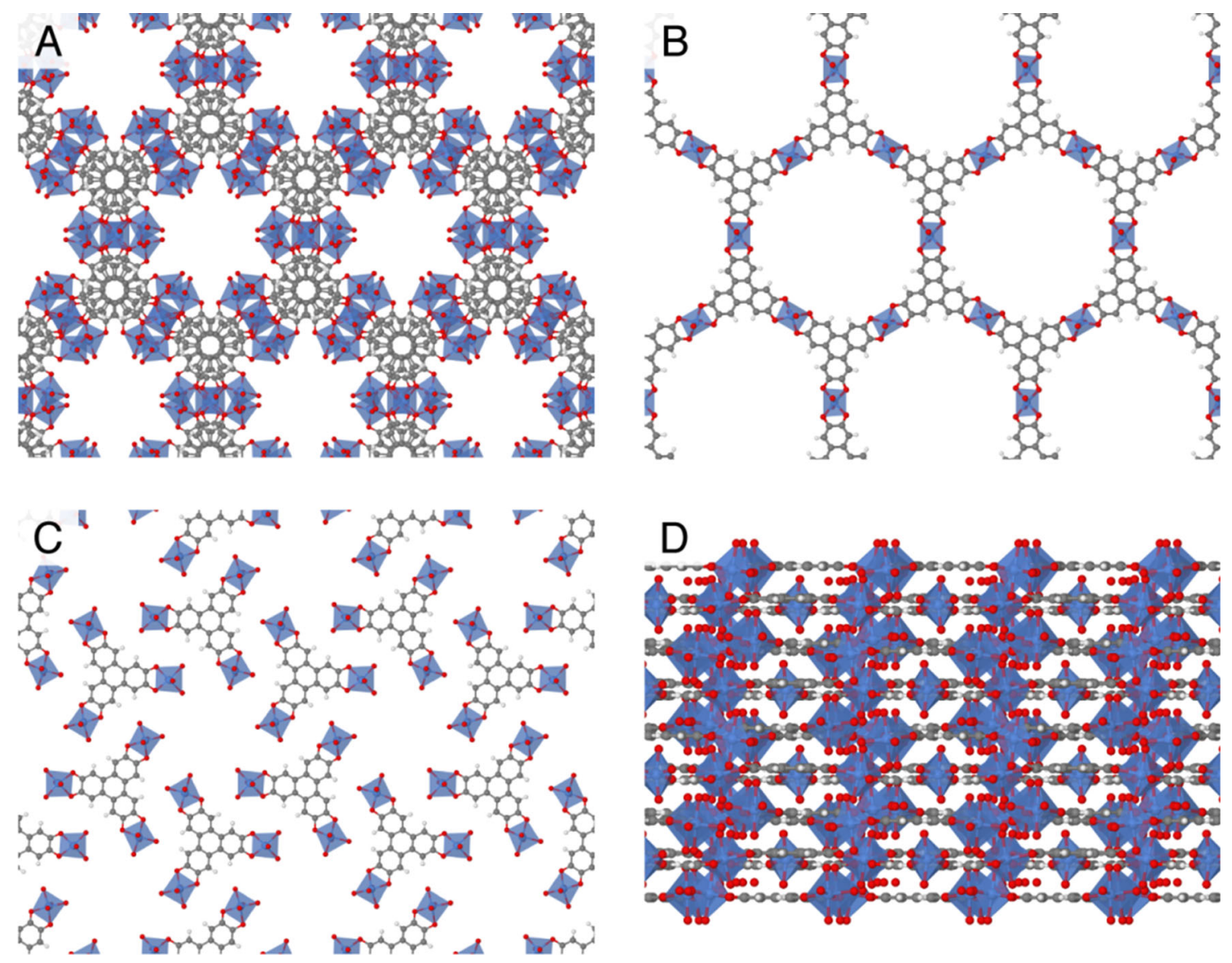

Fig. 1 Structure of M-CATs. View along the $c$ axis of the entire structure $\mathbf{a}$, of the continuous layer $\mathbf{b}$ and of the discrete units layer $\mathbf{c}$. View of the stacked layers along the $a$ axis $\mathbf{d}$. Blue polyhedra, red, grey and white represent the metal ion $\mathrm{M}^{2+}$, oxygen, carbon and hydrogen respectively

ligands complete the octahedral coordination sphere of the metal ions. In this continuous layer, the formal charge of the redox-active HHTP linker is -3 , corresponding to the semiquinonate form for each of the three catecholate rings. The overall structure formula of this type of layer is $\mathrm{M}_{3}(\mathrm{HHTP})_{2}\left(\mathrm{H}_{2} \mathrm{O}\right)_{6}$. In the second layer, only one linker molecule, as well as four water ligands, coordinate the metal ion (Fig. 1c). The charge of HHTP in these discrete $\mathrm{M}_{3}(\mathrm{HHTP})\left(\mathrm{H}_{2} \mathrm{O}\right)_{12}$ complexes is -6 . The two types of layers are stacked in an eclipsed fashion with the HHTP molecules in each layer rotated $60^{\circ}$ with respect to each other (Fig. 1a, d). The layers are held together by two types of non-covalent interactions: hydrogen bond interactions between the axial water ligands of the discrete units and the oxygen atoms of the HHTP in the adjacent continuous layers, as well as $\pi-\pi$ interactions between the large conjugated aromatic ring structures of the linker molecules. The overall stacked structure exhibits one-dimensional pores with a diameter of approximately $12 \AA$.

The present study focusses on the use of these M-CATs as catalysts for base catalyzed reactions; it thus assesses the wider applicability of deprotonated hydroxyl functionalities in MOFs as basic sites, beyond MOFs of the MOF-74 type. For the already reported materials $\mathrm{Ni}$ - and $\mathrm{Cu}-\mathrm{CAT}$, their catalytic activity in Knoevenagel condensation and Michael addition reactions is evaluated; new M-CATs are synthesized with $\mathrm{Mg}^{2+}, \mathrm{Ca}^{2+}, \mathrm{Sr}^{2+}, \mathrm{Ba}^{2+}$ and $\mathrm{Zn}^{2+}$ as the metal ion to investigate the influence of the metal ion on the catalytic activity of the materials.

\section{Materials and Methods}

\subsection{Materials}

Magnesium ethoxide and malononitrile were purchased from Fluka; barium nitrate, sodium tert-butoxide and benzaldehyde from Acros; toluene from VWR; ethyl cyanoacetate from Aldrich; copper(II) acetylacetonate and methyl vinyl ketone from Sigma-Aldrich; nickel acetate tetrahydrate from Janssen Chimica, calcium ethoxide and zinc acetylacetonate from ABCR; strontium nitrate from UCB and 2,3,6,7,10,11-hexahydroxytriphenylene from TCI. All chemicals were of the highest grade available and were used without further purification. 


\subsection{M-CAT Synthesis}

Synthesis procedures for the M-CATs were based on a scaled up version of the procedure reported by Hmadeh et al. [8]. In each case, $350 \mathrm{mg}$ of HHTP $(1.08 \mathrm{mmol})$ and two equivalents of the metal salt (nickel acetate, zinc acetylacetonate or copper(II) acetylacetonate) were added to $200 \mathrm{ml}$ of water in a glass Schott flask. The mixtures were sonicated for $20 \mathrm{~min}$ and placed overnight in an oven at $85{ }^{\circ} \mathrm{C}$. The M-CATs were then removed from the synthesis solution via centrifugation, washed three times with water and three times with acetone, and dried in air.

PXRD screening of synthesis conditions using different alkaline earth metal salts showed that the best results were obtained using magnesium and calcium ethoxide salts. Nitrate salts were used for barium and strontium, combined with the addition of sodium tert-butoxide (six equivalents) to the synthesis mixture.

Prior to their use as catalyst, the materials were vacuum pretreated overnight at $85^{\circ} \mathrm{C}$ to remove all the residual solvent molecules from the structure.

\subsection{M-CAT Characterization}

The crystallinity and structure of the synthesized materials were confirmed via powder X-ray diffraction (PXRD). Reflection patterns were recorded on a STOE STADI MP

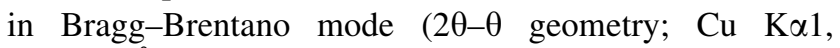
$1.54060 \AA$ ) using a linear position-sensitive detector.

$\mathrm{N}_{2}$ sorption measurements were performed on a Micromeritics 3Flex surface analyzer at $77 \mathrm{~K}$. Prior to measurements, the samples were activated under vacuum at $85{ }^{\circ} \mathrm{C}$.

Scanning electron microscopy micrographs were recorded using a Philips XL30 FEG after coating with Au, coupled with energy-dispersive X-ray spectroscopy (EDX) at $15 \mathrm{kV}$.

Fourier transform infrared spectra (FTIR) of the materials were recorded using Varian 670 FTIR Imaging microscope equipped with a Ge Slide-on ATR module.

Thermogravimetric analyses of the materials were performed using a TA Instruments Q500 thermogravimetric analyzer. Experiments were conducted under $\mathrm{O}_{2}$-flow with a linear heating ramp of $5{ }^{\circ} \mathrm{C} / \mathrm{min}$.

\subsection{Catalytic Testing}

In a typical reaction, $1 \mathrm{mmol}$ of each reactant was dissolved in $2 \mathrm{ml}$ of toluene. The mixture was added to $25 \mathrm{mg}$ of activated catalyst. The reaction mixture was heated to $70{ }^{\circ} \mathrm{C}$ and aliquots of sample were removed for analysis at set intervals. The identity of the reaction products was verified by GC-MS (Agilent 6890 gas chromatograph, equipped with a HP-5MS column, coupled to a 5973 MSD mass spectrometer) and the product yields were determined via GC-analysis.

\section{Results and Discussion}

\subsection{Material Synthesis and Characterization}

The Ni-CAT material was synthesized according to a scaled up variant of the literature procedure; the synthesis of the $\mathrm{Cu}$-CAT deviated from this procedure as another metal source was used, i.e. copper(II) acetylacetonate rather than copper(II) trifluoroacetylacetonate. Similarly, zinc acetylacetonate was used as the metal source for the synthesis of Zn-CAT. The formation of the correct materials was confirmed via powder X-ray diffraction (PXRD, Fig. 2). Electron micrographs (Fig. S1, Supporting information) show that the $\mathrm{Zn}$-CAT particles have a needle shaped morphology, analogous to that of the reported $\mathrm{Ni}$ CAT material.

Different metal salts, with different anions, were screened in attempts to synthesize the alkaline earth metal CATs. The best results were obtained when using metal

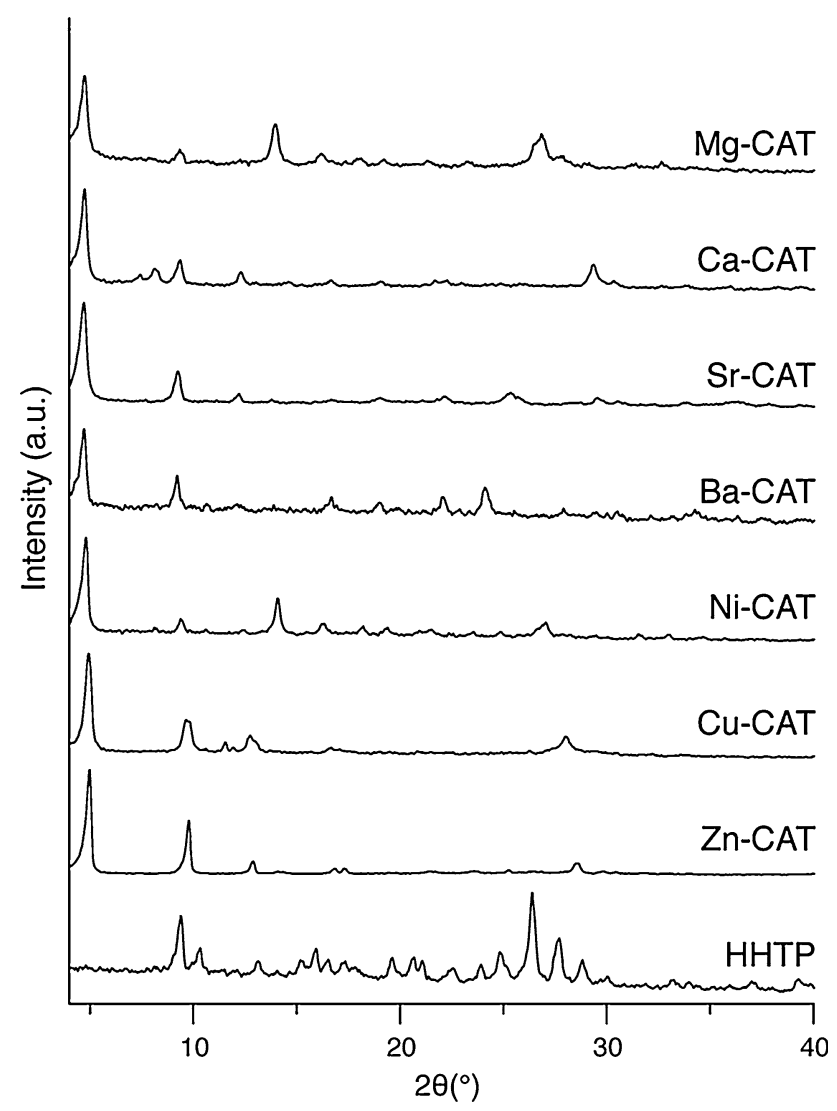

Fig. 2 PXRD patterns for the M-CAT materials and the pure HHTP linker 
alkoxides as a metal source. Both $\mathrm{Mg}$ - and $\mathrm{Ca}$-CAT were synthesized using their ethoxide salt, and Sr- and Ba-CAT could be synthesized using their nitrate salt and adding sodium tert-butoxide to the synthesis mixture. The presence of these alkoxides in the synthesis mixture could have two effects. Firstly, the alkoxides increase the $p H$ and facilitate the deprotonation of the HHTP linker molecule. Secondly, it is known that increasing the $p H$ of a mixture containing catechol compounds increases the rate of semiquinonate formation, which is in fact an oxidative process using air as the oxidant [10]. In this way, the increased $\mathrm{pH}$ could facilitate the formation of the continuous layers in the M-CAT structure. PXRD confirmed the formation of the correct phases. For all divalent cations used, the intense first reflection at $4.7^{\circ} 2 \theta$ confirms that the arrangement of the linkers and layers is similar as in the hexagonal structure of the Ni-CAT material; the characteristic $d$-spacing derived from the 100 reflection is $1.88 \mathrm{~nm}$ for Ni-CAT (Fig. 2). Electron microscopy of Caand Ba-CAT (Fig. S1) shows that the particles again have a needle shaped morphology, yet for Ca-CAT, the aspect ratio of the needles is considerably lower and the particle sizes are much less uniform.

The extent of linker deprotonation was evaluated with FTIR for Sr-, Ba- and Zn-CAT (Fig. S2). The FTIR spectrum of the parent HHTP linker shows absorption bands at 3456 and $1270 \mathrm{~cm}^{-1}$ which correspond to $\mathrm{O}-\mathrm{H}$ and $\mathrm{Ar}-\mathrm{O}$ stretch vibrations respectively. These absorption bands are absent in the spectra of the M-CAT materials, confirming the deprotonation of the linker molecules.

The porosity of the materials was evaluated using nitrogen physisorption at $77 \mathrm{~K}$ (Table 1, Fig. S3 \& S4). The BET surface areas for the different materials show large variations, beyond those that are to be expected due to the different atomic weights of the metal ions. The value of $413 \mathrm{~m}^{2} / \mathrm{g}$ measured for Ni-CAT (Table 1, entry 5) corresponds well with the reported value of $425 \mathrm{~m}^{2} / \mathrm{g}$ [8]. The rather low value of $78 \mathrm{~m}^{2} / \mathrm{g}$ for Cu-CAT (entry 6) can unfortunately not be compared to literature as no value was reported by Hmadeh et al. An intermediate value was recorded for the Zn-CAT material (entry 7). A broad

Table 1 BET surface areas for the different M-CAT materials

\begin{tabular}{llc}
\hline & $M$ & BET surface area $\left(\mathrm{m}^{2} / \mathrm{g}\right)$ \\
\hline 1 & $\mathrm{Mg}$ & 52 \\
2 & $\mathrm{Ca}$ & 63 \\
3 & $\mathrm{Sr}$ & 447 \\
4 & $\mathrm{Ba}$ & 119 \\
5 & $\mathrm{Ni}$ & 413 \\
6 & $\mathrm{Cu}$ & 78 \\
7 & $\mathrm{Zn}$ & 292 \\
\hline
\end{tabular}

variation of the surface areas was also observed for the alkaline earth metal CATs. Interestingly, the lightest metal ions, i.e. $\mathrm{Mg}^{2+}$ and $\mathrm{Ca}^{2+}$ (entries $1 \& 2$ ), show the lowest surface areas of the complete M-CAT series, whereas the highest surface area, $447 \mathrm{~m}^{2} / \mathrm{g}$, was observed for the $\mathrm{Sr}$ CAT (entry 3). In view of the monodimensional pore system of these materials, it is obvious that even a minor amount of stacking errors or fault planes can strongly affect the accessible pore volumes.

To assess whether these strong differences in surface area, measured using $\mathrm{N}_{2}$-physisorption, could influence the activity of the materials, a liquid phase adsorption experiment of benzaldehyde in heptane was conducted using both the high surface area Sr-CAT and the low surface area $\mathrm{Ca}$ CAT (Fig. 3). The much larger adsorption capacity of SrCAT compared to that of Ca-CAT reflects the differences observed in the $\mathrm{N}_{2}$-physisorption measurement and indicates that the M-CATs featuring a large surface area indeed feature a more open pore structure with a higher number of accessible active sites. When comparing the catalytic activities for these materials, the large differences in surface areas should therefore be kept in mind.

\subsection{Catalytic Testing}

The catalytic activity of the different M-CATs was evaluated using three different reactions. The first two reactions are Knoevenagel condensation reactions in which malononitrile (MN) and ethyl cyanoacetate (ECA) are coupled to benzaldehyde (BA) and water is expelled. The results for these reactions are summarized in Table 2. There are marked differences in the catalytic activities observed for the different metal ions. For the condensation of BA and $\mathrm{MN}$, after $2 \mathrm{~h}$ an increasing activity is observed

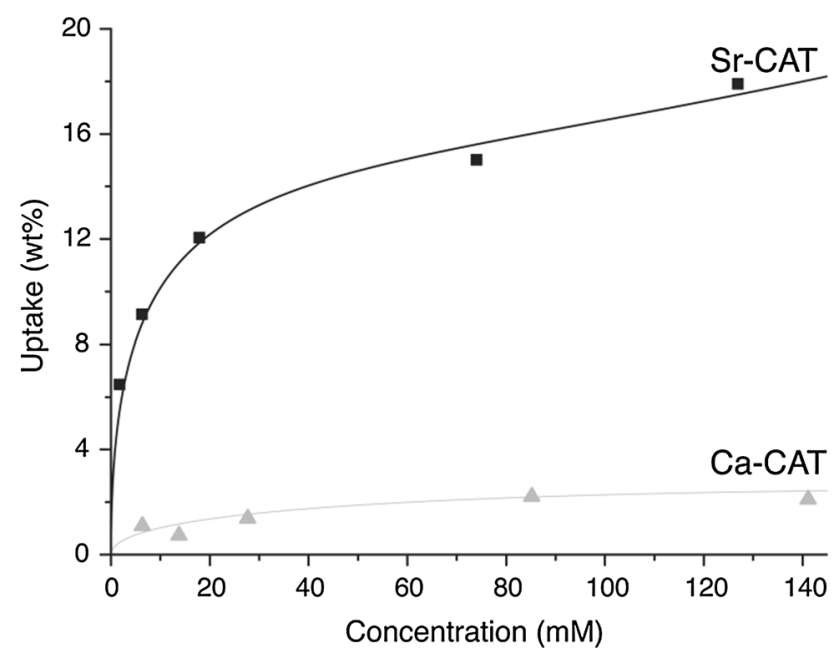

Fig. 3 Single compound adsorption for benzaldehyde on Sr- and CaCAT measured out of heptane in batch mode at $298 \mathrm{~K}$ : uptake (wt\%) as a function of equilibrium phase concentration 
for $\mathrm{Mg} \approx \mathrm{Ca}<\mathrm{Zn} \approx \mathrm{Ba}<\mathrm{Sr}<\mathrm{Ni} \approx \mathrm{Cu}$. After $24 \mathrm{~h}$, the observed differences are less clear, yet the Ba- (84\%) and Ni-CAT (90\%) (Table 2 entries 4-6) give the highest conversions. Increasing the reaction time to $48 \mathrm{~h}$ further increases the conversion to 93 and $94 \%$ for $\mathrm{Ba}-$ and NiCAT respectively. For the condensation of $\mathrm{BA}$ and ECA, the observed conversions are markedly lower than for the reaction with $\mathrm{MN}$. This is likely due to the lower acidity of ECA, with a $p \mathrm{~K}_{\mathrm{a}}$ of 13.1 in DMSO, compared to $\mathrm{MN}$ which has a $p \mathrm{~K}_{\mathrm{a}}$ of 11.1 in DMSO. The differences in catalytic activity of the different M-CATs in this reaction are much less pronounced, with only the Ni-variant standing out after $2 \mathrm{~h}$ of reaction with a BA conversion of $9 \%$ (entry 5). After $24 \mathrm{~h}$, the conversions observed for BaCAT $(23 \%)$ and Cu-CAT (22\%) surpass these of Ni-CAT (14\%). Overall, activity for the M-CATs, based on the conversion after $24 \mathrm{~h}$ of reaction, appears to increase in the series $\mathrm{Ca} \approx \mathrm{Mg}<\mathrm{Zn}<\mathrm{Ni} \approx \mathrm{Sr}<\mathrm{Cu} \approx \mathrm{Ba}$.

Although the activity series appears to be quite random at first glance, the data are much better understood when discriminating between the transition metal ions $\mathrm{Ni}^{2+}$ and $\mathrm{Cu}^{2+}$, and the non-transition metal ions $\mathrm{Mg}^{2+}, \mathrm{Ca}^{2+}, \mathrm{Sr}^{2+}$, $\mathrm{Ba}^{2+}$ and $\mathrm{Zn}^{2+}$. The rationale behind this discrimination is the fact that the $d$-orbitals of the transition metal ions may contribute in the bonding of e.g. reactant molecules or reaction intermediates, whereas the alkaline earth and $\mathrm{Zn}^{2+}$ ions are expected to interact solely via $\sigma$-donation from the ligand to the metal ion.

When focusing on the non-transition metal ions, the following activity series are observed after $2 \mathrm{~h}$ of reaction: $\mathrm{Mg}^{2+} \approx \mathrm{Ca}^{2+}<\mathrm{Zn}^{2+} \approx \mathrm{Ba}^{2+}<\mathrm{Sr}^{2+}$ for $\mathrm{MN}+\mathrm{BA}$ and $\mathrm{Mg}^{2+} \approx \mathrm{Ca}^{2+}<\mathrm{Zn}^{2+} \approx \mathrm{Ba}^{2+}<\mathrm{Sr}^{2+}$ for $\mathrm{ECA}+$ $\mathrm{BA}\left(\mathrm{Mg}^{2+} \approx \mathrm{Ca}^{2+}<\mathrm{Zn}^{2+}<\mathrm{Sr}^{2+}<\mathrm{Ba}^{2+}\right.$ after $24 \mathrm{~h}$ of reaction). It appears that the specific surface area of the different M-CATs is an important determining factor for their catalytic activity. The low surface area materials,
Mg- and Ca-CAT, are indeed consistently the weakest catalysts. This however is not the only factor at play. The catalytic activity of Ba-CAT in the Knoevenagel condensation reactions is for example equal to or greater than that of $\mathrm{Zn}-\mathrm{CAT}$, which is contradictory to the expectations based on the specific surface areas. A likely explanation for this observation may be found in the electronegativities of the different metal ions and their impact on the basicity of the materials, similar as for base catalytic activities of alkaline earth metal oxides [11]. As the catecholate linker coordinates to the metal ion, part of the electron density of the basic deprotonated hydroxyl oxygen atoms will be transferred to the metal ions, effectively decreasing the basicity of these sites. As the electronegativity of the metal ion gets lower, less electron density is expected to be transferred to the metal ion and more negative charge will remain on the basic oxygen site. Use of a less electronegative metal ion should therefore result in a more basic material. The electronegativities for the different metal ions used in this work are listed in Table 3 [12-14]. Ranking the different metal ions based on their absolute electronegativities, i.e. $\mathrm{Mg}^{2+}>\mathrm{Ca}^{2+}>\mathrm{Zn}^{2+}>\mathrm{Sr}^{2+}>$ $\mathrm{Ba}^{2+}$ (or $\mathrm{Zn}^{2+}>\mathrm{Mg}^{2+}>\mathrm{Ca}^{2+}>\mathrm{Sr}^{2+}>\mathrm{Ba}^{2+}$ in the case of Sanderson electronegativities) indeed yields a similar yet inverse trend to that observed in catalytic activities for the Knoevenagel condensation reactions. Additionally, calculation of the partial charges on the catecholate oxygen atoms in the material using Sanderson electronegativities and the principle of electronegativity equalization [14, 15], indeed shows a more negative charge for the materials built up with low electronegative metal ions such as $\mathrm{Ba}^{2+}$ of $\mathrm{Sr}^{2+}$. Furthermore, these calculations show that the partial charges on the catecholate oxygen atoms are systematically larger for the layers consisting of discrete units compared to the continuous layers. This can be attributed to the higher oxidation state, hence lower electron density, of
Table 2 Conversion

$(X)$ measured after 2 and $24 \mathrm{~h}$ of reaction for the different M-CAT materials in

Knoevenagel condensation and Michael addition reactions

\begin{tabular}{|c|c|c|c|c|c|c|c|}
\hline & \multirow[t]{3}{*}{$M$} & \multirow{2}{*}{\multicolumn{2}{|c|}{$\frac{\text { Knoevenagel condensation }}{\mathrm{MN}+\mathrm{BA}}$}} & \multirow{2}{*}{\multicolumn{2}{|c|}{$\frac{\text { Knoevenagel condensation }}{\mathrm{ECA}+\mathrm{BA}}$}} & \multirow{2}{*}{\multicolumn{2}{|c|}{$\frac{\text { Michael addition }}{\mathrm{ECA}+\mathrm{MVK}}$}} \\
\hline & & & & & & & \\
\hline & & $X_{2 \mathrm{~h}}(\%)$ & $X_{24 \mathrm{~h}}(\%)$ & $X_{2 \mathrm{~h}}(\%)$ & $X_{24 \mathrm{~h}}(\%)$ & $X_{2 \mathrm{~h}}(\%)$ & $X_{24 \mathrm{~h}}(\%)$ \\
\hline 1 & $\mathrm{Mg}$ & 8 & 66 & 2 & 7 & 15 & 26 \\
\hline 2 & $\mathrm{Ca}$ & 10 & 62 & 2 & 6 & 14 & 33 \\
\hline 3 & $\mathrm{Sr}$ & 32 & 77 & 5 & 15 & 40 & 74 \\
\hline 4 & $\mathrm{Ba}$ & 23 & 84 & 4 & 23 & 38 & 62 \\
\hline 5 & $\mathrm{Ni}$ & 44 & 90 & 9 & 14 & 12 & 39 \\
\hline 6 & $\mathrm{Cu}$ & 45 & 80 & 4 & 22 & 5 & 13 \\
\hline 7 & $\mathrm{Zn}$ & 22 & 75 & 4 & 9 & 20 & 45 \\
\hline 8 & Blank & 3 & 6 & 0 & 0 & 0 & 0 \\
\hline
\end{tabular}

Reaction conditions: $1 \mathrm{mmol}$ of each reactant; $2 \mathrm{ml}$ of solvent, $343 \mathrm{~K}, 25 \mathrm{mg}$ of M-CAT material $M N$ malononitrile, $B A$ benzaldehyde, $E C A$ ethyl cyanoacetate, $M V K$ methyl vinyl ketone 
Table 3 Absolute and Sanderson electronegativities of the different metal ions [12-14] and partial charges on the catecholate oxygen atoms calculated form the Sanderson electronegativities [15]

\begin{tabular}{llllll}
\hline & $M$ & Absolute & Sanderson & \multicolumn{2}{c}{ Partial charge of catecholate O } \\
\cline { 5 - 5 } & & electronegativity $(\mathrm{eV})$ & Electronegativity & Discrete layer & Continuous layer \\
\hline 1 & $\mathrm{Mg}^{2+}$ & 47.59 & 0.683 & -0.38 & -0.33 \\
2 & $\mathrm{Ca}^{2+}$ & 31.39 & 0.721 & -0.37 & -0.32 \\
3 & $\mathrm{Sr}^{2+}$ & 27.30 & 0.946 & -0.35 & -0.31 \\
4 & $\mathrm{Ba}^{2+}$ & 23.76 & 1.318 & -0.33 & -0.30 \\
5 & $\mathrm{Ni}^{2+}$ & 26.67 & 1.940 & -0.30 & -0.28 \\
6 & $\mathrm{Cu}^{2+}$ & 28.56 & 1.980 & -0.29 & -0.28 \\
7 & $\mathrm{Zn}^{2+}$ & 28.84 & 2.223 & -0.28 & -0.27 \\
\hline
\end{tabular}

the semiquinonate linker molecules in the continuous layers. Overall, a higher basicity of the Ba-CAT compared to the $\mathrm{Zn}$-CAT, despite the differences in surface areas, could account for the similar reaction rates observed in these reactions. The Sr-CAT however shows the best results due to the combination of a high specific surface area and a relatively low electronegativity of the associated cation.

To investigate whether similar activity trends could be observed in other base catalyzed reactions, all M-CAT materials were also applied in a third reaction, the Michael addition of ethyl cyanoacetate to methyl vinyl ketone (Table 2). Among the non-transition metal ions, a similar activity trend emerges after $2 \mathrm{~h}$ of reaction, i.e. $\mathrm{Ca}^{2+} \approx \mathrm{Mg}^{2+}<\mathrm{Zn}^{2+}<\mathrm{Ba}^{2+} \approx \mathrm{Sr}^{2+}$. After $24 \mathrm{~h}$ of reaction, the differences between the catalysts are more clear, with $\mathrm{Mg}^{2+}<\mathrm{Ca}^{2+}<\mathrm{Zn}^{2+}<\mathrm{Ba}^{2+}<\mathrm{Sr}^{2+}$. The overall catalytic activity of the materials can again be ascribed to the combined effects of the surface area and the electronegativity of the metal ions. The former relates to the number of accessible active sites, whereas the latter influences the strength of the individual sites.

The transition metal CATs, i.e. Ni- and $\mathrm{Cu}-\mathrm{CAT}$, show a high catalytic activity in the Knoevenagel condensations (entries 5 \& 6 in Table 2), especially when taking into account the low specific surface area of $\mathrm{Cu}-\mathrm{CAT}$. This observation is in line with the findings on MOF-74-type MOFs, where the $\mathrm{Ni}_{2}$ dobdc and $\mathrm{Cu}_{2}$ dobdc materials showed higher catalytic activities for these reactions than the $\mathrm{Mg}$ or $\mathrm{Zn}$ versions of this material (dobdc $=2,5$ dioxido-1,4-benzenedicarboxylate) [6]. Comparing the catalytic activity of e.g. $\mathrm{Ni}_{2}$ dobdc with that of $\mathrm{Ni}-\mathrm{CAT}$ in the reaction of $\mathrm{BA}+\mathrm{MN}$, shows that after $2 \mathrm{~h}, \mathrm{Ni}_{2}$ dobdc reached a little over 2 turnovers whereas close to 5 turnovers were recorded for Ni-CAT, based in both cases on the total amount of Ni-sites. This could indicate that the larger number of phenolate/catecholate oxygen atoms per metal ion in the framework of the Ni-CAT material indeed leads to a higher catalytic activity for the M-CAT materials. In the Michael addition however, the catalytic activities of Ni- and Cu-CAT are strikingly lower than those of $\mathrm{Ba}^{-}$or Sr-CAT. No clear rationalization for this phenomenon can be given. Possibly, it may arise from a difference in reaction mechanisms between the Knoevenagel condensation and Michael addition reactions, which leads to differences in rates between the transition- and non-transition metal ion CATs for the different reactions.

\subsection{Catalyst Heterogeneity and Reusability}

The catalyst heterogeneity was investigated using hot filtration tests for the Ni-, Sr- and Ba-CATs. The results of these tests are summarized in Fig. 4. After removal of the catalyst, the reaction rate drops to that of the blank reaction, confirming the heterogeneous nature of the catalyst. Furthermore, reuse of the Ba-CAT in three consecutive runs shows that the materials can be readily recycled (Fig. 5) and PXRD measurements before and after reaction (Fig. 6) show that the materials are stable under reaction conditions.

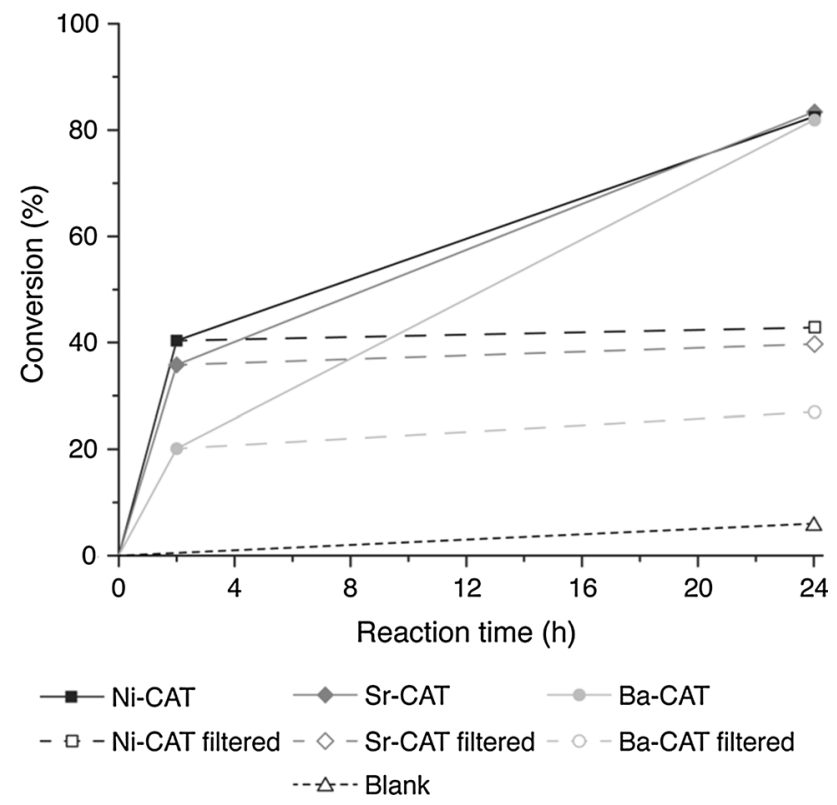

Fig. 4 Hot filtration experiments for the Knoevenagel condensation of malononitrile and benzaldehyde catalyzed by Ni-, Sr- and Ba-CAT. Filtration after $2 \mathrm{~h}$ 


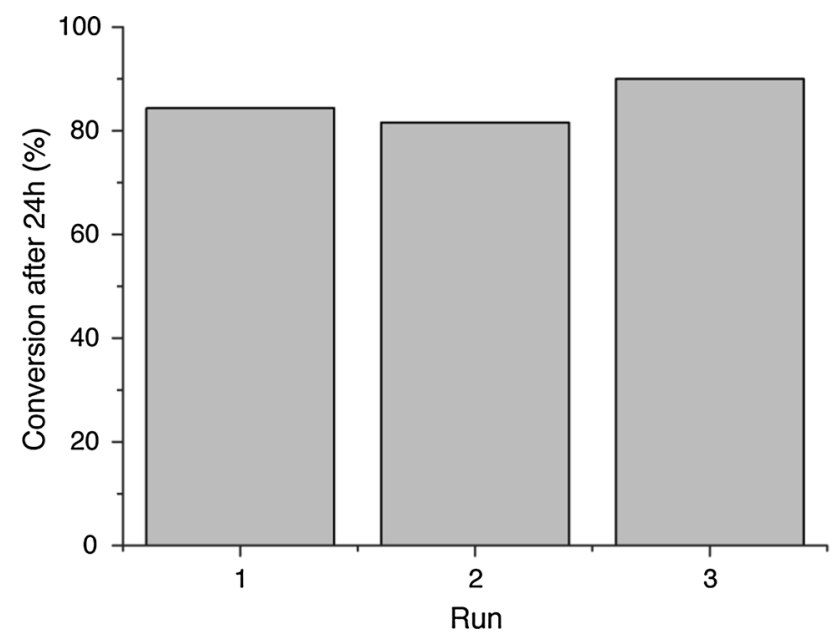

Fig. 5 Recycle test for the Ba-CAT catalyst in the Knoevenagel condensation of benzaldehyde and malononitrile in toluene at $70{ }^{\circ} \mathrm{C}$

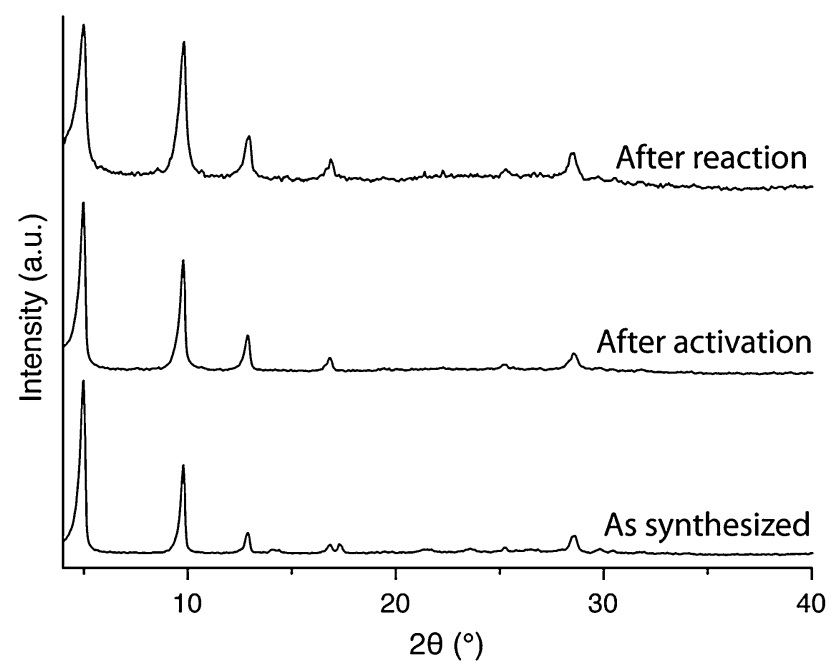

Fig. 6 PXRD patterns for the Zn-CAT material as synthesized, after activation and after reaction

\section{Conclusion}

$\mathrm{N}$-functionalized linker molecules are widely accepted as building blocks for the incorporation of basic sites in metal-organic frameworks. The limited basicity of such groups may however restrict the widespread applicability of these materials as catalysts. In line with the known base catalytic activity of metal-dioxidoterephthalate MOFs of the CPO-27 type, the catalytic activity of metal catecholate frameworks in Knoevenagel condensation and Michael addition reactions confirms that deprotonated hydroxyl groups form an alternative structural motive that introduces potent basic sites in MOFs.

The synthesis of alkaline earth metal variants of these M-CAT materials furthermore showed that the catalytic activity of the materials, with a large number of basic sites, is highly tunable. Two major parameters control the overall catalytic activity of the materials: the specific surface area of the materials relates to the number of active sites, and the electronegativity of the metal ion determines the strength of the individual sites.

Acknowledgments The authors are grateful to the Belgian Federal Government for support in the IAP project 07/05 Functional Supramolecular Systems, to K. U. Leuven for the Methusalem CASAS grant, and to FWO Vlaanderen for research project funding G.0453.09 and G.0486.12. P.V. is grateful for a fellowship from FWO Vlaanderen.

\section{References}

1. Gascon J, Aktay U, Hernandez-Alonso MD, van Klink GPM, Kapteijn F (2009) Amino-based metal-organic frameworks as stable, highly active basic catalysts. J Catal 261(1):75-87. doi:10. 1016/j.jcat.2008.11.010

2. Kasinathan P, Seo YK, Shim KE, Hwang YK, Lee UH, Hwang DW, Hong DY, Halligudi SB, Chang JS (2011) Effect of diamine in amine-functionalized MIL-101 for knoevenagel condensation. Bull Korean Chem Soc 32(6):2073-2075. doi:10.5012/bkcs.2011. 32.6.2073

3. Savonnet M, Aguado S, Ravon U, Bazer-Bachi D, Lecocq V, Bats N, Pinel C, Farrusseng D (2009) Solvent free base catalysis and transesterification over basic functionalised metal-organic frameworks. Green Chem 11(11):1729-1732

4. Tan Y, Fu Z, Zhang J (2011) A layered amino-functionalized zinc-terephthalate metal organic framework: structure, characterization and catalytic performance for Knoevenagel condensation. Inorg Chem Commun 14(12):1966-1970. doi:10.1016/j. inoche.2011.09.022

5. Valvekens P, Jonckheere D, De Baerdemaeker T, Kubarev AV, Vandichel M, Hemelsoet K, Waroquier M, Van Speybroeck V, Smolders E, Depla D, Roeffaers MBJ, De Vos D (2014) Base catalytic activity of alkaline earth MOFs: a (micro)spectroscopic study of active site formation by the controlled transformation of structural anions. Chem Sci 5(11):4517-4524. doi:10.1039/ c4sc01731e

6. Valvekens P, Vandichel M, Waroquier M, Van Speybroeck V, De Vos D (2014) Metal-dioxidoterephthalate MOFs of the MOF-74 type: microporous basic catalysts with well-defined active sites. J Catal 317:1-10. doi:10.1016/j.jcat.2014.06.006

7. Song J, Wu L, Zhou B, Zhou H, Fan H, Yang Y, Meng Q, Han B (2015) A new porous $\mathrm{Zr}$-containing catalyst with a phenate group: an efficient catalyst for the catalytic transfer hydrogenation of ethyl levulinate to [gamma]-valerolactone. Green Chem. doi:10.1039/c4gc02104e

8. Hmadeh M, Lu Z, Liu Z, Gándara F, Furukawa H, Wan S, Augustyn V, Chang R, Liao L, Zhou F, Perre E, Ozolins V, Suenaga K, Duan X, Dunn B, Yamamto Y, Terasaki O, Yaghi OM (2012) New porous crystals of extended metal-catecholates. Chem Mater 24(18):3511-3513. doi:10.1021/cm301194a

9. Yaghi OM, Gandara-Barragan F, Wan S, Lu Z (2012) Preparation of metal-catecholate frameworks. 09.08.2012

10. Lebedev AV, Ivanova MV, Timoshin AA, Ruuge EK (2007) Effect of group II metal cations on catecholate oxidation. Chem Phys Chem 8(12):1863-1869. doi:10.1002/cphc.200700296

11. Tanabe K, Saito K (1974) The conversion of benzaldehyde into benzyl benzoate with alkaline earth metal oxide catalysts. J Catal 35(2):247-255. doi:10.1016/0021-9517(74)90203-6 
12. Pearson RG (1988) Absolute electronegativity and hardness: application to inorganic chemistry. Inorg Chem 27(4):734-740. doi:10.1021/ic00277a030

13. Atkins $\mathrm{P}$, Overton T, Rourke J, Weller M, Armstrong F, Hagerman M (2010) Shriver and Atkins' Inorganic Chemistry, 5th edn. Oxford University Press, Great Britain
14. Sanderson RT (1988) Principles of electronegativity Part I. Gen nat J Chem Educ 65(2):112. doi:10.1021/ed065p112

15. Sanderson RT (1976) Chemical bonds and bond energy. Academic Press, New York 